\title{
La residencia fiscal en Colombia, problemáticas de la normativa actual y propuestas desde la óptica internacional ${ }^{1}$
}

\section{Tax residency in Colombian Tax Law, issues arising from the domestic legislation and proposals from an international perspective}

\author{
ÓSCAR MUNÉVAR ${ }^{2}$
}

\section{Resumen}

La residencia fiscal es un criterio de conexión entre un Estado y los sujetos pasivos del impuesto sobre la renta. En Colombia la principal consecuencia de ser considerado residente fiscal es la obligación de tributar por las rentas de fuente mundial y su patrimonio localizado dentro y fuera del país. La norma tributaria colombiana no establece una definición del concepto "residente", pues se limita a señalar los criterios a partir de los cuales un sujeto pasivo debe ser considerado residente en el país, para lo cual en algunas ocasiones utiliza el concepto de "domicilio" o "nacional". Este artículo pretende analizar las implicaciones fiscales del concepto de residencia en Colombia, tanto para las personas naturales como para las sociedades y demás entidades, así como las principales problemáticas y sus posibles soluciones.

\begin{abstract}
The tax residence is a nexus between a State and income tax taxpayers. Colombian tax residents are subject to an unlimited liability to tax (i.e. are subject to taxes over their worldwide source income and equity). However, Colombian Tax Law rather than provide a definition of tax residence determines the criterion pursuant to which a subject would be tax resident. This article aims to analy-
\end{abstract}

${ }^{1}$ Éste artículo fue desarrollado con base en el trabajo de grado en Derecho "La residencia fiscal en Colombia y el ámbito subjetivo de aplicación de los Convenios de Doble Imposición Internacional" presentado en 2015 y dirigido por el profesor Mauricio Marín Elizalde.

${ }^{2}$ Abogado de la Universidad Externado de Colombia. Abogado Sénior de la sublínea de Impuestos Internacionales en EY Colombia y Miembro Alumni del Observatorio Iberoamericano de Tributación Internacional - OITI. Las opiniones expresadas en este escrito son personales y no comprometen a ninguna de las instituciones con las que el autor está vinculado. Para citar el artículo: Munévar, Ó. (2016). "La residencia fiscal en Colombia, problemáticas de la normativa actual y propuestas desde la óptica internacional", en Revista de Derecho Fiscal n. ${ }^{\circ}$ 9, Bogotá: Universidad Externado de Colombia.pp. 63-76. DOI: http://dx.doi.org/10.18601/16926722.n9.05 
ze and provide conclusions regarding the tax consequences that may arise from the concept of residence as set forth by the law in force, for both physical persons and corporations, as well as the main issues arising from its regulation and provide alternative solutions to these.

Palabras clave: Residencia fiscal, personas naturales, personas jurídicas, nacionales colombianos, sociedades nacionales, sociedades extranjeras, sede efectiva de administración.

Keywords: Tax residence, physical persons, corporations, Colombian nationals, tax residence of corporations, foreign corporations, Place of effective management.

Sumario: Introducción. I. La residencia fiscal. 1. Implicaciones tributarias derivadas de la residencia fiscal en Colombia. II. La residencia fiscal en Colombia de las personas naturales. 1. Permanencia física en Colombia. 1.1. Conflictos de residencia generados por la aplicación del criterio de permanencia en el país. 1.1.1. Concurrencia de diversas categorías en el mismo período gravable. 1.1.2. Efectos en el segundo año cuando se completa la residencia. 1.1.3. Períodos de trecientos sesenta y cinco días sin sujeción al período gravable. 2. Relación con el servicio exterior del Estado colombiano. 3. Nacionales colombianos. 3.1. Nacionales que no acrediten ser residentes fiscales en otro país o tengan residencia fiscal en un "paraíso fiscal”. 4. Causales de exclusión aplicables a los nacionales colombianos. III. Residencia fiscal en Colombia de las sociedades o entidades. 1. Sede de administración efectiva.
1.1. Causales de exclusión del criterio de sede de administración efectiva. 1.1.1. Sociedades que coticen en la Bolsa de Valores de Colombia y/o en una bolsa de reconocida idoneidad internacional, o sus subordinadas, filiales o subsidiarias consolidadas a nivel contable. 1.1.2. Sociedades o entidades del exterior cuyos ingresos de fuente de la jurisdicción, donde esté constituida la sociedad o entidad del exterior sean iguales o superiores al $80 \%$ de sus ingresos totales. 2. Domicilio principal de la sociedad. 3. Lugar de incorporación de la sociedad. Referencias.

\section{Introducción}

La residencia fiscal es un criterio de conexión entre la potestad tributaria de un Estado y los sujetos pasivos del impuesto sobre la renta y complementarios. Sin embargo, la norma tributaria colombiana no establece una definición de residencia fiscal, sino que se limita a señalar los criterios que determinan cuándo una persona natural, o una sociedad o entidad extranjera, es considerada residente fiscal en Colombia.

El Estatuto Tributario establece las normas en materia de residencia fiscal en sus artículos $10 .^{\circ}$ (para el caso de las personas naturales), y 12-1 y 21 (para el caso de las sociedades y entidades). Estas normas fueron objeto de modificaciones en las dos últimas reformas tributarias ${ }^{3}$ con el propósito de lograr mayor claridad y certeza en la determinación de la residencia fiscal, así como alinear la normatividad doméstica colombiana con los estándares internacionales -utilizando como guía el Modelo de Convenio para Eliminar la Doble Imposición de la OCDE (Zarama y Zarama, 2013).

${ }^{3}$ Leyes 1607 de 2012 y 1739 de 2014. 
Sin embargo, como se analiza en el presente artículo, la aplicación de esa nueva normatividad genera no pocas dificultades prácticas y diferencias en su interpretación.

\section{La residencia fiscal}

\section{Implicaciones tributarias derivadas de la residencia fiscal en Colombia}

En Colombia la condición de residente fiscal tiene para los sujetos, como principal consecuencia, el surgimiento de la obligación de tributar por las rentas de fuente mundial así como por el patrimonio que posea dentro y fuera del país ${ }^{4}$ durante el o los periodos gravables en que sean residentes fiscales colombianos.

En contraposición, los sujetos considerados no residentes fiscales están obligados a tributar en Colombia exclusivamente por las rentas de fuente nacional ${ }^{5}$ y por el patrimonio que posean en el país ${ }^{6}$.

Adicionalmente, la condición de residente fiscal abre la posibilidad de aplicar los beneficios derivados de los Convenios de Doble Imposición-CDI- pues, de conformidad con los artículos $1 .^{\circ}$ y $4 .^{\circ}$ de los CDI suscritos por Colombia, estos únicamente serán aplicables por aquellas personas consideradas residentes fiscales a partir de la legislación doméstica colombiana.

Por otra parte, la condición de residente fiscal determina las tarifas de retención en la fuente establecidas en los artículos 407 a 415 del Estatuto Tributario y aplicables únicamente a sujetos considerados no residentes fiscales en Colombia. En virtud del artículo
592 del Estatuto Tributario la retención en la fuente practicada conforme a los artículos 407 a 411 del Estatuto Tributario será el impuesto final para el no residente.

La Ley 1739 del 2014 estableció hasta el 2018 tarifas diferenciales del impuesto sobre la renta para aquellas sociedades y entidades extranjeras sin residencia fiscal en el país que perciban ingresos de fuente colombiana no atribuibles a un establecimiento permanente.

En razón a la precaria técnica legislativa no era claro si la introducción de esas tarifas diferenciales modificaba de manera implícita las tarifas de retención en la fuente señaladas en los artículos 407 a 411 , las cuales, como se indicó, constituyen el impuesto final para el no residente. Sin embargo, la Administración Tributaria consideró, de manera acertada, que las tarifas de retención en la fuente señaladas en los artículos 407 a 411 no fueron modificadas, pues para que eso fuese procedente, el legislador debió haber señalado expresamente esa modificación (Oficios DIAN 11676 y 12343 de 2015).

\section{La residencia fiscal en Colombia de las personas naturales}

De conformidad con el artículo $10{ }^{\circ}$ del Estatuto Tributario, se consideraran residentes fiscales aquellas personas naturales que cumplan con alguno de los siguientes criterios: 1. Que tengan permanencia física en Colombia; 2. Que tengan relación con el servicio exterior del Estado colombiano, o 3. Que tengan nacionalidad colombiana.

${ }^{4}$ Artículos 9. ${ }^{\circ}, 12$ y 261 del Estatuto Tributario.

${ }^{5}$ El artículo 24 del Estatuto Tributario establece el listado, no taxativo, de aquellos ingresos considerados por la normativa tributaria colombiana como ingresos de fuente colombiana.

${ }^{6}$ Artículos 265 y 266 del Estatuto Tributario. 


\section{Permanencia física en Colombia}

De conformidad con la normativa tributaria, sin importar si se trata de nacional colombiano o extranjero, una persona natural será considerada residente fiscal cuando permanezca en el país por más de ciento ochenta y tres días calendarios, continuos o discontinuos, dentro de un período cualquiera de trescientos sesenta y cinco días calendario consecutivos ${ }^{7}$. En caso de que el período de permanencia se cumpla en más de un año gravable, la persona natural será considerada residente fiscal a partir del segundo año gravable.

Es necesario tener en cuenta que la autoridad tributaria colombiana-DIAN-aclaró que para el conteo de los términos establecidos en el artículo $10{ }^{\circ}$ del Estatuto Tributario únicamente se tendrán en cuenta los hechos ocurridos a partir del $1 .^{\circ}$ de enero de 2013 (Oficio DIAN 000648 de 2014), dejando claro que a aquellas personas que hayan permanecido en Colombia durante el año 2012 les será aplicable la normativa anterior para dicho período gravable.

Además, la norma señala que para efectos del conteo de los ciento ochenta y tres días deben tomarse en cuenta los días de entrada y de salida del país, situación que no estaba establecida de forma expresa en la normatividad anterior y que, por tanto, generaba diferencias de interpretación y aplicación.

Sin embargo, con respecto al conteo del término de trescientos sesenta y cinco días dentro de los cuales habrán de cumplirse los ciento ochenta y tres días de permanencia, únicamente se señala que este será un período cualquiera de días calendario consecutivo, redacción que, como se explicará, puede dar lugar a diversas interpretaciones con variadas consecuencias.

\subsection{Conflictos de residencia generados por la aplicación del criterio de permanencia en el país}

A pesar de que la nueva normativa fue introducida con el propósito de otorgar más claridad y certeza en la determinación de la residencia fiscal, la aplicación del criterio de permanencia puede llevar a generar situaciones de doble residencia, las cuales no tendrán solución, salvo que sea aplicable un $\mathrm{CDI}^{8}$.

Adicionalmente, una interpretación exegética de la norma, que parece ser la que ha adoptado la Administración Tributaria en su doctrina oficial, crea situaciones poco favorables para el contribuyente.

Los conflictos generados por la aplicación del criterio de permanencia en el país han sido agrupados en las siguientes categorías: (i) Concurrencia de diversas categorías en el mismo período gravable; (ii) Efectos en el segundo año cuando se completa la residencia, y (iii) Períodos de trescientos sesenta y cinco días sin sujeción al año gravable (Gutiérrez Argüello, 2015).

Sin embargo, consideramos que una lectura sistemática de la normatividad tributaria vigente puede dar solución a los dos primeros conflictos señalados por la autora.

\subsubsection{Concurrencia de diversas categorías en el mismo período gravable}

La interpretación de Gutiérrez Argüello es que en un mismo período fiscal pueden pre-

\footnotetext{
${ }^{7}$ Artículo $10{ }^{\circ}$, numeral 1 del Estatuto Tributario.

${ }^{8}$ El Artículo 4. ${ }^{\circ}$ de los CDI suscritos por Colombia establece reglas para dirimir los conflictos de doble residencia, las cuales serán aplicables, en principio, a personas residentes en los Estados con los cuales Colombia tiene suscrito un CDI.
} 
sentarse las siguientes categorías para una persona natural: (i) No sujeción en Colombia; (ii) No residente, y (iii) Residente fiscal.

De acuerdo con la redacción de la norma la autora considera que la persona natural estaría obligada a tributar sobre las rentas de fuente mundial generadas durante todo el período gravable, a pesar de que antes de su primer ingreso no debería ser considerada sujeto pasivo del impuesto en Colombia, salvo que perciba ingresos de fuente colombiana. Adicionalmente, señala que en el entre tanto la persona cumpla los ciento ochenta y tres días no es aún residente fiscal en Colombia y por tanto deben aplicársele las normas relativas a los no residentes (Gutiérrez Argüello, 2015).

Por su parte, la Administración Tributaria señaló que:

El contribuyente del impuesto sobre la renta obligado a declarar deberá hacerlo por el período completo dada la inescindibilidad del mismo, y no por fracciones de año (...) Una cosa es la contabilización de los días para concretar la residencia y otra distinta es la obligación de declarar por todo el período (Oficio DIAN 007234 de 2015) (cursiva fuera de texto).

Sin embargo, tanto la interpretación de Gutiérrez Argüello como la de la Administración desconocen la existencia de casos en los cuales la normatividad tributaria establece períodos impositivos inferiores a un año calendario: de conformidad con el numeral 2 del artículo 1.6.1.5.7. del Decreto 1625 de 2016 (el cual compila el art. 1. ${ }^{\circ}$ del Decreto 187 de 1975), para los extranjeros el período gravable comenzará o terminará en las respectivas fechas de llegada y de salida al país respectivamente.

En consecuencia, y de conformidad con la norma arriba citada, el período gravable de un extranjero que cumpla los ciento ochenta y tres días de permanencia en Colombia se inicia el primer día de entrada al país y termina el día de salida o el 31 de diciembre de ese año gravable. Así las cosas, y en contraposición a la argumentación de la autoridad tributaria, existen eventos en los que una persona natural obligada a declarar podrá hacerlo por un período gravable inferior al período gravable ordinario.

Por todo lo anterior, la concurrencia de diversas categorías no debe generar una situación adversa para los extranjeros, pues a estos les es aplicable un período gravable inferior al año calendario, razón por la cual únicamente deben tributar por las rentas de fuente mundial percibidas con posterioridad a su primer día de ingreso al país.

No obstante, como se señaló, la solución que parece prever la norma es descartada por la administración tributaria, razón por la cual la eventual aplicación por un contribuyente podrá dar lugar a discusiones.

Por otra parte, es importante resaltar que el período gravable especial señalado en el artículo 1.6.1.5.7. del Decreto 1625 de 2016 genera un tratamiento diferencial injustificado para aquellos nacionales colombianos que están en la misma situación que los extranjeros que ingresen al país, puesto que, incluso si el nacional colombiano ingresa al país a mitad del año gravable, para él el período gravable corresponde a la totalidad del año calendario $\mathrm{y}$, por tanto, debe incluir en su declaración aquellas rentas de fuente extranjera obtenidas con anterioridad a su ingreso al país.

\subsubsection{Efectos en el segundo año cuando se completa la residencia}

De acuerdo con el numeral 1 del artículo $10 .^{\circ}$ del Estatuto Tributario, cuando los 
ciento ochenta y tres días comprenden dos períodos gravables la persona natural es considerada residente fiscal en el segundo año gravable.

Sin embargo, varias interpretaciones, entre ellas la de la autora citada, consideran que ello trae como consecuencia que la persona natural continúe siendo considerada residente fiscal en Colombia con posterioridad a su salida del país.

La administración tributaria comparte esta interpretación señalando expresamente que:

[...] si los 183 días calendario de permanencia continua o discontinua (incluidos los días de entrada y salida del país) abarcan dos períodos fiscales y en el segundo año o período fiscal permaneció solo un (1) día de los 183 exigidos por la ley, se considera residente en Colombia a partir del segundo año o período gravable (Oficio DIAN 000657 de 2013) (cursiva fuera de texto).

Sin embargo, como se indicó, el numeral 2 del artículo 1.6.1.5.7. del Decreto 1625 de 2016 establece que el período gravable de los extranjeros termina el día de salida del país, por lo que, en virtud de una interpretación sistemática de las normas tributarias, cuando el extranjero permanezca en el segundo año o período gravable solo un día de los ciento ochenta y tres exigidos por el artículo $10{ }^{\circ}$ del Estatuto Tributario, su período gravable será de un día.

En ese sentido el artículo 6..$^{\circ}$ del Decreto 1651 de 1961 establece, para las personas que se ausenten del país con carácter definitivo, la obligación de presentar declaración de renta y patrimonio antes de su salida. Dicha norma presume que la salida del país de los extranjeros siempre será de carácter definitivo.
Así las cosas, un extranjero que salga del país en el segundo día calendario del año y haya cumplido en el primer día los ciento ochenta y tres días requeridos por la norma tributaria en materia de residencia debe ser considerado residente fiscal únicamente por el período gravable especial que le aplica en virtud del artículo 1.6.1.5.7. del Decreto 1625 de 2016 y del Decreto 1651 de 1961.

Finalmente, como se señaló en el punto anterior, la aplicación de estas normas genera un trato diferencial injustificado para el nacional colombiano, quien en principio es considerado residente fiscal por la totalidad del período gravable, aun cuando haya permanecido en el país por tan solo un día de dicho año.

\subsubsection{Períodos de trecientos sesenta y cinco} días sin sujeción al período gravable

Finalmente, de la lectura del artículo $10 .^{\circ}$ del ET no es posible inferir que a partir de la primera entrada al país en un período gravable se empiece a contar un único período de trecientos sesenta y cinco días. En interpretación de Gutiérrez Argüello, el conteo del período de trecientos sesenta y cinco días podrá hacerse a partir de cada día de entrada al país, en caso de que existan varios.

Sin embargo, la norma señala que será un período cualquiera de trecientos sesenta y cinco días calendario consecutivos, por lo que, en principio, nada impide a la administración tributaria realizar los conteos de esos períodos a partir de cualquier día posterior a la fecha de entrada al país de la persona natural.

Esas consecuencias indeseadas pueden solucionarse sujetando el período de trecientos 
sesenta y cinco días al año calendario, como lo establece la normatividad española ${ }^{9}$.

Por otra parte, legislaciones como la de los Estados Unidos de América establecen una fórmula ponderada para aquellos períodos de permanencia que superan un año calendario, denominado test de presencia sustancial (substantial presence test), el cual utiliza para la suma de los días de permanencia una fórmula ponderada con los siguientes porcentajes (EYGM Limited, 2015):

Año actual $100 \%$

Primer año anterior $33.33 \%$

Segundo año anterior $16.67 \%$

Es preciso anotar que la legislación americana trae ciertas excepciones para efectos del conteo de esos días, las cuales no exponemos aquí por su extensión y por exceder el objeto del presente documento.

\section{Relación con el servicio exterior del Estado colombiano}

También son consideradas residentes fiscales en Colombia aquellas personas naturales que por su relación con el servicio exterior del Estado colombiano o con personas que prestan sus servicios en este, y en virtud de la Convención de Viena sobre Relaciones Diplomáticas de 1961 y de la Convención de Viena sobre Relaciones Consulares de 1963, estén exentos de imposición sobre la renta en el país en donde se encuentren en misión.

En ese sentido, el numeral 2 del artículo $10 .^{\circ}$ del Estatuto Tributario no establece como requisito que la persona trabaje por un tiempo mínimo para la misión a efectos de ser considerada como residente fiscal en Colombia (Zarama y Zarama, 2013).

\section{Nacionales colombianos}

Finalmente, el numeral 3 del artículo $10{ }^{\circ}$ del Estatuto Tributario señala que serán considerados residentes fiscales en Colombia aquellas personas naturales que sean nacionales colombianos ${ }^{10}$ y que además cumplan alguna de las siguientes condiciones:

a) Que su cónyuge o compañero permanente no separado legalmente o los hijos dependientes menores de edad, tengan residencia fiscal en el país; o,

b) Que el cincuenta por ciento $(50 \%)$ o más de sus ingresos sean de fuente nacional; o,

c) Que el cincuenta por ciento $(50 \%)$ o más de sus bienes sean administrados en el país; o,

d) Que el cincuenta por ciento (50\%) o más de sus activos se entiendan poseídos en el país.

El literal a aclaró el concepto de "familia”, establecido de forma genérica por la anterior legislación como "el núcleo humano más próximo al contribuyente" (Oficio DIAN 005 de 2003).

No obstante, para la aplicación de esa condición hay que tener en cuenta que el Consejo de Estado ha ampliado la noción de familia, dejando de lado la definición tradicional que tenía, y reconociendo por tanto la conformación de familia en parejas del mismo sexo (Sentencia CE-1048 de 2014).

Así las cosas, y en aras de una interpretación sistemática, ha de entenderse que el literal a del numeral 3 del artículo $10 .^{\circ}$ del

${ }^{9}$ Artículo 9. ${ }^{\circ}$ de la Ley 35 de 2006 de España (Ley del Impuesto de Renta de las Personas Físicas - LIRPF).

${ }^{10}$ Artículo 96 de la Constitución Política de Colombia. 
Estatuto Tributario le es igualmente aplicable a las parejas del mismo sexo que cumplan con los requisitos para ser considerados compañeros permanentes.

Por su parte, las condiciones señaladas por los ordinales b, c y d del numeral $3 \mathrm{del}$ artículo $10 .^{\circ}$ del Estatuto Tributario sustituyen el concepto de "asiento principal de los negocios" y establecen la forma como se determinará el concepto de centro de intereses económicos de la persona natural colombiana, para efectos de la determinación de su residencia fiscal.

Esas condiciones conforman lo que en la doctrina internacional se conoce como el "centro de intereses vitales" de la persona natural, concepto que pretende tener en cuenta las relaciones económicas y personales con el Estado para efectos de determinar su residencia fiscal. El centro de intereses vitales ha de determinarse con base en la vida familiar de la persona, así como en el lugar en que desarrolle sus intereses y actividades sociales (Lang, 2014).

El centro de intereses vitales que establece la normativa tributaria colombiana aplica únicamente para los nacionales colombianos pese a que, como se observó, ese concepto ha sido desarrollado por la doctrina internacional para que sea aplicable, de manera indistinta, a nacionales y extranjeros. Así, consideramos que la norma establece un trato diferencial injustificado para los nacionales colombianos, pues un extranjero no será considerado residente fiscal en Colombia aun en el evento en que se encuentre en la misma situación que un nacional colombiano al que le aplique alguno de estos criterios.

\subsection{Nacionales que no acrediten ser residentes fiscales en otro país o tengan residencia fiscal en un "paraíso fiscal"}

Adicionalmente, de conformidad con el ordinal e del numeral 3 del artículo $10 .^{\circ}$ del Estatuto Tributario y el parágrafo del mismo artículo, cuando un nacional colombiano tenga su residencia fiscal en otro país debe acreditarlo ante la Dirección de Impuestos y Aduanas Nacionales con un certificado de residencia fiscal emitido por la autoridad competente del país en el cual esté residenciado. En el evento en que el nacional colombiano no aporte esa certificación será considerado residente fiscal en Colombia.

Por otra parte, también serán considerados residentes fiscales aquellos nacionales colombianos que tengan su residencia fiscal en alguna de las jurisdicciones calificadas como paraísos fiscales ${ }^{11}$.

La norma no establece limitación temporal con respecto a la calificación de residente fiscal para aquel nacional colombiano residente en un paraíso fiscal, por lo que no es claro si el análisis debe hacerse en cada período gravable, o si por el contrario es una calificación permanente.

Pese a la falta de regulación, creemos que una interpretación adecuada debe considerar residentes fiscales únicamente por un período gravable a aquellos nacionales colombianos residenciados en paraísos fiscales, razón por la cual anualmente se debe comprobar si continúan residenciados en uno de ellos.

Sin embargo, para evitar cualquier discusión interpretativa, la norma en cuestión

\footnotetext{
${ }^{11}$ Para efectos tributarios el listado de jurisdicciones consideradas paraísos fiscales para los colombianos está incluido en el artículo 1.2.2.5.1 del Decreto 1625 de 2016 (el cual compila el art. 1. ${ }^{\circ}$ del Dcto. 1966 de 2014, modificado por el art. 1. ${ }^{\circ}$ del Dcto. 2095 de 2014).
} 
debe incluir una limitación temporal, como la incluye la norma tributaria española en virtud de la cual los nacionales españoles residentes en "paraísos fiscales" continúan siendo considerados residentes fiscales en España por el período fiscal en el cual se efectúe el cambio de residencia y los cuatro siguientes períodos impositivos ${ }^{12}$.

Los supuestos explicados en este acápite tienen como propósito contrarrestar la evasión fiscal, pues

... se establece un esquema bajo el cual el nacional colombiano será residente para efectos fiscales y tributará en Colombia sobre la renta mundial, así no permanezca, ni tenga actividad o familia en el país, salvo que [certifique que es residente fiscal] en otro país y ese país no sea considerado paraíso fiscal [por la normatividad colombiana] (Zarama y Zarama, 2013).

\section{Causales de exclusión aplicables a los nacionales colombianos}

El artículo 25 de la Ley 1739 de 2014 estableció que los nacionales colombianos no serán considerados residentes fiscales en Colombia cuando cumplan alguna de las siguientes condiciones:

i) Que el cincuenta por ciento (50\%) o más de sus ingresos anuales tengan su fuente en la jurisdicción en la cual tengan su domicilio.

ii) Que el cincuenta por ciento (50\%) o más de sus activos se encuentren localiza- dos en la jurisdicción en la cual tengan su domicilio.

Sin embargo, a la fecha no se ha establecido regulación alguna con relación a estas condiciones, ni es claro el procedimiento o las formalidades, pues no las establece la norma, para que los nacionales colombianos acrediten su cumplimiento.

Adicionalmente, la aplicación de esos dos supuestos genera oportunidades de planeación tributaria para aquellos nacionales colombianos que tengan la posibilidad de transferir la fuente de más del $50 \%$ de sus ingresos anuales o sus activos a la jurisdicción en la cual tengan su "domicilio".

Es importante señalar que, de acuerdo con la redacción de la norma, esas causales de exclusión pueden ser aplicadas aun en el evento en que el nacional colombiano esté "domiciliado" en un paraíso fiscal.

Finalmente, puede ser que debido a una precaria técnica legislativa, la normativa en cuestión haga referencia a la jurisdicción en la cual la persona natural nacional tenga su "domicilio"; sin embargo, no determina qué debe entenderse por tal (Zarama y Zarama, 2015).

\section{Residencia fiscal en Colombia de las sociedades 0 entidades}

Las personas jurídicas se consideran residentes fiscales en Colombia cuando, de acuerdo con las reglas establecidas en el Estatuto Tributario, son sociedades o entidades nacionales $^{13}$.

Desde la perspectiva de la legislación comercial, la nacionalidad de las personas ju-

${ }^{12}$ Artículo 8. ${ }^{\circ}$ de la Ley 35 de 2006 de España (Ley del Impuesto de Renta de las Personas Físicas - LIRPF)

${ }^{13}$ Artículos $12-1$ y 21 del Estatuto Tributario. 
rídicas tiene como propósito establecer el régimen jurídico aplicable a su formación y funcionamiento (Reyes Villamizar, 2011). Sin embargo, desde el punto de vista tributario, la nacionalidad de las sociedades o entidades determina la obligación de tributar por sus rentas de fuente mundial, o únicamente por sus rentas de fuente colombiana si son consideradas sociedades o entidades extranjeras ${ }^{14}$.

El artículo 12-1 del Estatuto Tributario señala los criterios de sede de administración efectiva (Place of Effective Management), domicilio principal y lugar de incorporación de la sociedad para efectos de determinar cuándo una sociedad es nacional colombiana.

\section{Sede de administración efectiva}

De conformidad con la norma tributaria, una sociedad o entidad tendrá como sede de administración efectiva aquel lugar en donde materialmente se toman las decisiones comerciales y de gestión, decisivas y necesarias, para llevar a cabo las actividades de la sociedad o entidad como un todo ${ }^{15}$.

Esta redacción es muy similar, por no decir idéntica, a la definición del concepto de sede de dirección efectiva que incluye la Organización para la Cooperación y el Desarrollo Económico-OCDE- en el parágrafo 24 de los Comentarios al artículo 4 del Modelo de Convenio de la OCDE.

Sin embargo, la norma plantea una diferencia sustancial con el concepto de sede de dirección efectiva desarrollado por la OCDE, pues señala que:

Par. 1: Para determinar la sede efectiva de administración deben tenerse en cuenta todos los hechos y circunstancias que resulten pertinentes, en especial el relativo a los lugares donde los altos ejecutivos y administradores de la sociedad o entidad usualmente ejercen sus responsabilidades y se llevan a cabo las actividades diarias de la alta gerencia de la sociedad o entidad.

Pár. 2: No se considerará que una sociedad o entidad es nacional por el simple hecho de que su junta directiva se reúna en el territorio colombiano (cursiva fuera de texto).

Así las cosas, pese a que el concepto de sede de dirección efectiva desarrollado por la OCDE le da relevancia a las actividades que determinan el rumbo de la compañía, es decir, aquellas que en principio únicamente podrían ser tomadas por la junta directiva de la compañía, la norma colombiana señala que este factor no es determinante per se de la sede de dirección efectiva en Colombia, y le da prevalencia al lugar en que las decisiones del día a día son efectuadas.

No obstante, la incorporación de este concepto en la legislación nacional también trae consigo inconvenientes prácticos, pues las reglas domésticas que hacen referencia al concepto de sede de dirección efectiva son normas abiertas, es decir, requieren un análisis detallado de los hechos y circunstancias del caso en concreto (De Broe, 2009), lo cual, consideramos, genera inseguridad jurídica para el contribuyente, toda vez que le otorga a la Administración Tributaria y a los jueces una amplia discrecionalidad interpretativa.

Frente a la incertidumbre jurídica que puede generar tal discrecionalidad interpretati-

\footnotetext{
${ }^{14}$ Artículo 12 del Estatuto Tributario.

${ }^{15}$ Parágrafo 1. ${ }^{\circ}$ del Artículo 12-1 del Estatuto Tributario
} 
va, la Administración Tributaria debe seguir un procedimiento uniforme que garantice un trato justo e igualitario para todos los contribuyentes.

En consecuencia, será necesario esperar a que la jurisprudencia desarrolle el concepto de sede de administración efectiva, pues hasta la fecha la doctrina oficial de la Administración Tributaria no se ha pronunciado a fondo al respecto.

Sin embargo, teniendo en cuenta que el concepto de sede de administración efectiva ya tiene un desarrollo doctrinario internacional, su implementación en Colombia debe traer de la mano la introducción de un procedimiento que de manera unificada permita verificar todos los hechos y circunstancias pertinentes para determinar la residencia fiscal de la sociedad o entidad en el país.

En ese sentido se propone que, frente a una norma de sede de dirección efectiva abierta, como la de nuestra legislación interna, se analicen e identifiquen tres aspectos: (i) Las actividades de administración propias del mercado en el que se desenvuelve la sociedad (aspecto sustancial); (ii) Las personas que realmente desarrollan las actividades de administración (aspecto personal), y (iii) La localización del lugar en que desarrollan las actividades (aspecto territorial) (De Broe, 2009).

Un análisis juicioso y sistemático de los aspectos mencionados debe conducir, en principio, a un tratamiento igualitario para los sujetos a los que se les pretenda aplicar la norma de sede de administración efectiva, lo que a su vez otorga seguridad jurídica.

Sin embargo, ese no parece ser el camino seguido por la Administración Tributaria al considerar que "el texto legal no utiliza expresiones oscuras o confusas, siendo suficiente una interpretación gramatical para mostrar con claridad su sentido, conforme con el artículo 27 del Código Civil (Oficio DIAN 058445 de 2013).

Con este concepto la Administración Tributaria pretende aclarar, de manera poco certera, los términos "durante" y "materialmente" que incluye el artículo 12-1 del Estatuto Tributario, señalando que la expresión "durante" hace referencia a cualquier momento del período gravable, y para aclarar "materialmente" remite a la definición establecida por la Real Academia Española (Oficio DIAN 058445 de 2013).

Por otra parte, el Parágrafo 3 del artículo 12-1 del Estatuto Tributario señala que en aquellos casos en que se discuta la determinación de la sede de administración efectiva de una sociedad o entidad, esta decisión será tomada por el Comité de Fiscalización de la DIAN. No obstante, de conformidad con la estructura orgánica de la DIAN para el $1 .^{\circ}$ de julio de 2016 ese comité no existía ${ }^{16}$.

Adicionalmente, ni la normatividad ni la regulación expedida establecen los efectos temporales de la declaratoria de la sede de administración efectiva en Colombia. Tal declaratoria debe tener efectos únicamente en el año gravable para el cual se efectúa el análisis de las circunstancias, pues estas son condiciones de hecho que por su naturaleza pueden variar en el tiempo.

Así las cosas, la única limitación temporal en favor de una sociedad o entidad que no haya cumplido con sus obligaciones como residente fiscal en Colombia es el término de cinco años, contados a partir del venci-

${ }^{16}$ Disponible en [http://www.dian.gov.co/DIAN/12sobred.nsf/fc22bc5cf1ab7bfa05257030005c2 805/8a347d3ad512be9a052574eb005cf1f6?OpenDocument], consultada el 2 de agosto de 2016. 
miento de plazo para declarar, para que la Administración Tributaria profiera liquidación de aforo ${ }^{17}$.

\subsection{Causales de exclusión del criterio de sede de administración efectiva}

La Ley 1739 de 2014 adicionó los parágrafos 5 y 6 al artículo 12-1 del ET, por medio de los cuales se establecieron las siguientes causales cuyo cumplimiento excluye la determinación de sede efectiva de administración en Colombia:

1.1.1. Sociedades que coticen en la Bolsa de Valores de Colombia o en una bolsa de reconocida idoneidad internacional, o sus subordinadas, filiales o subsidiarias consolidadas a nivel contable

Pese a que la norma es clara frente a qué tipo de sociedades aplica esta exclusión, ata su cumplimiento a que la sociedad matriz cotice en la Bolsa de Valores de Colombia o en una bolsa de reconocida idoneidad internacional de acuerdo con resolución que expida la DIAN.

Recientemente, la DIAN, mediante la Resolución n. ${ }^{\circ} 57$ del 27 de julio de 2016 listó las bolsas de valores que considera de "reconocida idoneidad" para efectos de la aplicación del artículo 12-1 del Estatuto Tributario.

\subsubsection{Sociedades o entidades del exterior} cuyos ingresos de fuente de la jurisdicción donde esté constituida la sociedad o entidad del exterior sean iguales o superiores al $80 \%$ de sus ingresos totales
Esta causal de exclusión implica una dificultad para su aplicación práctica, pues establece la necesidad de que el $80 \%$ de los ingresos de la sociedad sean de fuente de la jurisdicción en donde esté constituida.

La norma tributaria colombiana únicamente establece un listado enunciativo de aquellos ingresos considerados de fuente colombiana ${ }^{18} \mathrm{y}$ un listado taxativo de ingresos que no se consideran de fuente nacional ${ }^{19}$.

Así, para efectos de determinar la fuente de los ingresos es necesario considerar la normativa tributaria del país en que está constituida la sociedad (Zarama y Zarama, 2015). En ese sentido, para aplicar esa norma o desvirtuar la posición del contribuyente, la Administración Tributaria colombiana debe remitirse a la normatividad del país correspondiente. Lo anterior genera una imposibilidad para que el funcionario correspondiente aplique la norma pues, de acuerdo con el principio de legalidad que gobierna la función pública, únicamente podrá actuar dando aplicación a la ley vigente en Colombia.

Por otra parte, la norma señala la siguiente regla para el cálculo del $80 \%$ de los ingresos de la sociedad que sean de fuente de la jurisdicción en donde está constituida:

Ingresos de fuente extranjera $\geq 80 \%$ de los ingresos totales de la sociedad

Para la determinación de ese porcentaje, dentro de los ingresos totales generados en el exterior, no se tendrán en cuenta las rentas pasivas.

De acuerdo con la redacción de la norma, las rentas pasivas serán tomadas en cuenta para efectos de calcular el total de los ingre-

${ }^{17}$ Artículo 717 del Estatuto Tributario.

${ }^{18}$ Artículo 24 del Estatuto Tributario

${ }^{19}$ Artículo 25 del Estatuto Tributario. 
sos (de fuente nacional y de fuente extranjera) pero no para efectos de calcular el $80 \%$ de los ingresos de fuente extranjera (Zarama y Zarama, 2015).
A manera de ejemplo, los ingresos totales de una sociedad constituida en el exterior suman $\$ 100$, discriminados así:

\begin{tabular}{|l|l|c|}
\hline \multicolumn{1}{|c|}{ Fuente de los ingresos } & \multicolumn{1}{|c|}{ Tipo de ingreso } & Valor (\$) \\
\hline $\begin{array}{l}\text { Ingresos de fuente de la jurisdic- } \\
\text { ción en donde está constituida la } \\
\text { sociedad }\end{array}$ & Rentas pasivas (p. ej., dividendos) & 85 \\
\cline { 2 - 3 } & Rentas activas (p. ej., servicios) & 5 \\
\hline Ingresos de fuente colombiana & 10 \\
\hline
\end{tabular}

La ecuación correspondiente sería:

$\$ 90 \geq 80 \% *(\$ 5+\$ 10)$

(Entre los ingresos totales no se tendrán en cuenta los $\$ 85$ correspondientes a ingresos por dividendos -rentas pasivas-).

En consecuencia, los ingresos de fuente extranjera (\$90 según el ejemplo) serán superiores al $80 \%$ de los ingresos totales de la sociedad calculados según el artículo 12-1 del Estatuto Tributario (\$12).

\section{Domicilio principal de la sociedad}

El domicilio de una sociedad es la circunscripción territorial (municipio o distrito) que se pacta en sus estatutos sociales y en donde los asociados están llamados a ejercer sus derechos (Reyes Villamizar, 2011).

De esa forma, la decisión que tomen los socios para efectos comerciales de establecer el domicilio principal de la sociedad en Colombia tendrá como consecuencia, desde el punto de vista tributario, la calificación de la sociedad como residente fiscal colombiano.
En relación con este criterio no hay discusión pues se trata de un criterio de fácil verificación que solo implica remisión al certificado de existencia y representación legal expedido por la Cámara de Comercio correspondiente para conocer el domicilio principal de la sociedad en cuestión.

\section{Lugar de incorporación de la sociedad}

De conformidad con el numeral segundo del artículo 12-1 del Estatuto Tributario también son consideradas residentes fiscales aquellas sociedades constituidas en Colombia de acuerdo con las leyes vigentes en el país.

El principio subyacente al criterio de incorporación como factor determinante de la residencia de las personas jurídicas radica en el hecho de que la personalidad jurídica es un atributo concedido por las leyes del Estado bajo el cual fueron incorporadas (Behrens, 2009).

Como se observa, el criterio del lugar de incorporación de la sociedad es puramente formal, y tradicionalmente ha sido usado por las legislaciones tributarias para efectos de determinar la residencia fiscal de las personas jurídicas. 


\section{Referencias}

Behrens, Peter (2009). "A comparative analysis of connecting factors used for the determination of proper law of Companies", en Guglielmo Maisto (ed.). Residence of Companies under Tax Treaties and EC Law, The Hague, IBFD.

De Broe, Luc (2009). "Corporate tax residence in civil law jurisdictions", en Guglielmo Maisto (ed.). Residence of Companies under Tax Treaties and EC Law, The Hague, IBFD.

EY (2015). Worldwide personal tax guide, Income tax, social security and immigration, 2015-2016, EYGM Limited.

Gutiérrez Argüello, Myriam Stella (febrero de 2015). Algunos conflictos de la regla de residencia y del régimen de tributación de las personas naturales, Estudios de Derecho Tributario, Derecho Aduanero y Comercio Exterior.

Lang, Michael (2014). Introducción al derecho de los convenios para evitar la doble imposición, Diego Quiñones (trad.), Bogotá, IBFD y Editorial Temis.

OCDE (2014). Model Tax Convention on Income and on Capital: Condensed Version 2014, París, ocDE Publishing.

Reyes Villamizar, Francisco (2011). Derecho Societario, t. I, Bogotá, Editorial Temis.

Zarama Vásquez, Fernando y Camilo Zarama Martínez (2013). Reforma tributaria comentada. Ley 1607 de 2012, Bogotá, Legis.

Zarama Vásquez, Fernando y Camilo Zarama Martínez (2015). Reforma tributaria comentada. Ley 1739 de 2014, Bogotá, Legis. 